\title{
Research Article \\ Existence of Solutions for a Nonlinear Elliptic Equation with General Flux Term
}

\author{
Hee Chul Pak \\ Department of Applied Mathematics, Dankook University, Cheonan, Chungnam 330-714, Republic of Korea \\ Correspondence should be addressed to Hee Chul Pak, hpak@dankook.ac.kr \\ Received 25 September 2010; Revised 29 January 2011; Accepted 27 February 2011 \\ Academic Editor: D. R. Sahu \\ Copyright (C) 2011 Hee Chul Pak. This is an open access article distributed under the Creative \\ Commons Attribution License, which permits unrestricted use, distribution, and reproduction in \\ any medium, provided the original work is properly cited. \\ We prove the existence of solutions for an elliptic partial differential equation having more \\ general flux term than either $p$-Laplacian or flux term of the Leray-Lions type conditions: \\ $-\sum_{j=1}^{n}\left(\partial / \partial x_{j}\right)\left(\alpha\left(\left|u_{x_{j}}\right|\right) / u_{x_{j}}\right)=f$. Brouwer's fixed point theorem is one of the fundamental tools \\ of the proof.
}

\section{Introduction}

We are concerned with problems of partial differential equations such as a nonlinear elliptic equation

$$
-\nabla \cdot \mathbf{J}=f,
$$

which contains the flux term $\mathbf{J}$. The flux term $\mathbf{J}$ is a vector field that explains a movement of some physical contents $u$ such as temperature, chemical potential, electrostatic potential, or fluid flows. Physical observations tell us in general that $\mathbf{J}$ depends on $u$ and approximately on its gradient at each point $x$, that is, $\mathrm{J}=\mathrm{J}(x, \nabla, u(x))$. For linear cases, one can simply represent $\mathbf{J}$ as $\mathbf{J}=c \nabla u$ (on isotropic medium) or $\mathbf{J}=A \nabla u$ with a square matrix $A$ (on an isotropic medium). But for nonlinear cases, the situation can be much more complicated. One of the common assumptions is that $\mathbf{J}=|\nabla u|^{p-2} \nabla u$ is to produce the $p$-Laplacian

$$
\Delta_{p} u:=\nabla \cdot|\nabla u|^{p-2} \nabla u
$$


Slightly more general conditions, for example, the Leray-Lions type conditions, might be placed on $\mathrm{J}$, but it is too good to be true that the flux term has those kinds of growth conditions in reality.

We prove the existence of solutions for the elliptic partial differential equation

$$
-\sum_{j=1}^{n} \frac{\partial}{\partial x_{j}}\left(\frac{\alpha\left(\left|u_{x_{j}}\right|\right)}{u_{x_{j}}}\right)=f .
$$

Galerkin's approximation method and Brouwer's fixed point theorem are employed for the proof.

We introduce a new function space which is designed to handle solutions of nonlinear equations (1.3). This space is arisen from a close look at the $L^{p}$-norm $\|f\|_{L^{p}}=\left(\int_{X}|f(x)|^{p} d \mu\right)^{1 / p}$ of the classical Lebesgue spaces $L^{p}(X), 1 \leq p<\infty$. It can be rewritten as

$$
\|f\|_{L^{p}}:=\alpha^{-1}\left(\int_{X} \alpha(|f(x)|) d \mu\right) \text {, with } \alpha(x):=x^{p} .
$$

Even though the positive-real-variable function $\alpha(x):=x^{p}$ has very beautiful and convenient algebraic and geometric properties, it also has some practical limitations to handle general nonlinear problems. The new space is devised to overcome these limitations without hurting the beauty of $L^{p}$-norm too much. Unfortunately the new space is only equipped with an inhomogeneous norm, and so it lacks the homogeneity property: $\|k f\|=|k|\|f\|$. However, for nonlinear problems such as (1.3), the homogeneity property may not be an essential factorwe try to explain that the new space accommodates the solutions of nonlinear problems without homogeneity.

Although this space is similar to the Orlicz spaces, we present a different approach of discovering the new spaces which generalize the space $L^{p}$.

\section{The Space $L_{\alpha}(X)$}

We introduce some terminologies to define the Lebesgue-type function spaces $L_{\alpha}(X)$. In the following, $(X, \mathfrak{M}, \mu)$ always represents a given measurable space.

\subsection{Hölder's Functions}

A pre-Hölder's function $\alpha: \mathbb{R}_{+} \rightarrow \mathbb{R}_{+}\left(\mathbb{R}_{+}=\{x \in \mathbb{R}: x \geq 0\}\right)$ is an absolutely continuous bijective function satisfying $\alpha(0)=0$. If there exists a pre-Hölder's function $\beta$ and $\lambda$ satisfying

$$
\alpha^{-1}(x) \beta^{-1}(x)=\lambda(x)
$$

and $c_{1} x<\lambda(x) \leq c_{2} x\left(x \in \mathbb{R}_{+}\right)$for some constants $1 \leq c_{1}<c_{2}$, then $\beta$ is called the conjugate (pre-Hölder's) function of $\alpha$ linked by $\lambda$. In the relation (2.1), the notations $\alpha^{-1}, \beta^{-1}$ are meant to be the inverse functions of $\alpha, \beta$, respectively. Examples of pre-Hölder's pairs are $(\alpha(x), \beta(x))=$ $\left(x^{p}, x^{q}\right),(p>1,1 / p+1 / q=1)$ with $\lambda(x)=x$ and $(\alpha(x), \beta(x))=\left(e^{x}-x-1,(1+x) \log (1+x)-x\right)$. 
In fact, for any Orlicz $N$-function $A$ together with complementary $N$-function $\tilde{A}$ and any positive constant $c \leq 1,(c A, c \tilde{A})$ is a pre-Hölder's pair with $\lambda(x)=(1 / c) A^{-1}(x) \tilde{A}^{-1}(x)$ (see page 264 in [1]).

Some basic identities for a pre-Hölder's pair $(\alpha, \beta)$ with respect to $\lambda$ are in order: for $\tilde{\alpha}:=\lambda \circ \alpha$, and $\tilde{\beta}:=\lambda \circ \beta$,

$$
\begin{gathered}
x=\beta\left(\frac{\lambda(x)}{\alpha^{-1}(x)}\right) \quad \text { or } \quad \alpha(x)=\beta\left(\frac{\tilde{\alpha}(x)}{x}\right), \\
x=\frac{\tilde{\alpha}(x)}{\beta^{-1}(\alpha(x))} \quad \text { or } \quad \frac{\tilde{\alpha}(x)}{x}=\beta^{-1} \circ \alpha(x)=\tilde{\beta}^{-1} \circ \tilde{\alpha}(x), \\
\tilde{\alpha}^{-1}(x)=\frac{x}{\tilde{\beta}^{-1}(x)}, \\
\frac{\beta^{-1}(x)}{\alpha^{\prime}\left(\alpha^{-1}(x)\right)}+\frac{\alpha^{-1}(x)}{\beta^{\prime}\left(\beta^{-1}(x)\right)}=\lambda^{\prime}(x), \\
\frac{y}{\alpha^{\prime}(x)}+\frac{x}{\beta^{\prime}(y)}=\lambda^{\prime}(\alpha(x)), \quad \text { for } y:=\frac{\tilde{\alpha}(x)}{x}, \\
\tilde{\alpha}^{\prime}(x)=\frac{\tilde{\alpha}(x)}{x}+\frac{\tilde{\alpha}(x)}{\tilde{\beta}^{\prime}(\tilde{\alpha}(x) / x)-x} .
\end{gathered}
$$

Remark 2.1. The conjugate identity (2.1) implicitly requires to have the following conditions:

$$
\lim _{x \rightarrow 0} \frac{\lambda(\alpha(x))}{x}=0, \quad \lim _{x \rightarrow \infty} \frac{\lambda(\alpha(x))}{x}=\infty .
$$

Let $\alpha$ be a given pre-Hölder's function. For every link-function $\lambda$ satisfying

$$
\lim _{x \rightarrow 0} \frac{\lambda(x)}{\alpha^{-1}(x)}=0, \quad \lim _{x \rightarrow \infty} \frac{\lambda(x)}{\alpha^{-1}(x)}=\infty
$$

there exists a conjugate function $\beta$ of $\alpha$ associated with $\lambda$.

In the following discussion, a function $\Phi$ represents the two-variable function on $\mathbb{R}_{+} \times$ $\mathbb{R}_{+}$defined by

$$
\Phi(x, y):=\alpha^{-1}(x) \beta^{-1}(y)
$$

provided that a pre-Hölder's pair $(\alpha, \beta)$ exists.

Definition 2.2. A pre-Hölder's function $\alpha: \mathbb{R}_{+} \rightarrow \mathbb{R}_{+}$together with the conjugate function $\beta$ for a link-function $\lambda$ is said to be a Hölder's function if for any positive constants $a, b>0$, there exist constants $\theta_{1}, \theta_{2}$ (depending on $a, b$ ) such that

$$
\theta_{1}+\theta_{2} \leq 1
$$


and that a comparable condition

$$
\Phi(x, y) \leq \theta_{1} \frac{a b}{\lambda \circ \alpha(a)} x+\theta_{2} \frac{a b}{\lambda \circ \beta(b)} y
$$

holds for all $(x, y) \in \mathbb{R}_{+} \times \mathbb{R}_{+}$.

The following proposition and the proof may illustrate that the comparable condition (2.12) is not farfetched.

Proposition 2.3. Let $\alpha$ be a convex pre-Hölder's function with the convex conjugate function $\beta$. Suppose that, for any $a, b \geq 0$, there are constants $p_{1}, p_{2}, q_{1}, q_{2}$ (depending on $a, b$ ) with $1 / p_{1}+1 / p_{2} \leq$ $1 \leq 1 / q_{1}+1 / q_{2}$ satisfying the slop conditions

$$
\begin{aligned}
& p_{1} \frac{\lambda \circ \alpha(a)}{a} \leq \alpha^{\prime}(a) \leq q_{1} \frac{\alpha(a)}{a} \\
& p_{2} \frac{\lambda \circ \beta(b)}{b} \leq \beta^{\prime}(b) \leq q_{2} \frac{\beta(b)}{b} .
\end{aligned}
$$

Then $\alpha$ is, in fact, a Hölder's function (so is $\beta$ ).

Proof. Indeed, the equation of the tangent plane of the graph of $\Phi$ at $(\bar{a}, \bar{b})$ reads

$$
\begin{aligned}
z & =\Phi_{x}(\bar{a}, \bar{b})(x-\bar{a})+\Phi_{y}(\bar{a}, \bar{b})(y-\bar{b})+\Phi(\bar{a}, \bar{b}) \\
& =\frac{\beta^{-1}(\bar{b})}{\alpha^{\prime}\left(\alpha^{-1}(\bar{a})\right)}(x-\bar{a})+\frac{\alpha^{-1}(\bar{a})}{\beta^{\prime}\left(\beta^{-1}(\bar{b})\right)}(y-\bar{b})+\alpha^{-1}(\bar{a}) \beta^{-1}(\bar{b}) \equiv T(x, y) .
\end{aligned}
$$

Then, for $\alpha^{-1}(\bar{a}):=a$ and $\beta^{-1}(\bar{b}):=b, T(x, y)$ can be rewritten as

$$
T(x, y)=\frac{b}{\alpha^{\prime}(a)} x+\frac{a}{\beta^{\prime}(b)} y+a b-\frac{b \alpha(a)}{\alpha^{\prime}(a)}-\frac{a \beta(b)}{\beta^{\prime}(b)} .
$$

From the slop conditions (2.13) together with the observation that

$$
\frac{b \alpha(a)}{\alpha^{\prime}(a)}+\frac{a \beta(b)}{\beta^{\prime}(b)} \geq \frac{1}{q_{1}} a b+\frac{1}{q_{2}} a b \geq a b,
$$

we have

$$
T(x, y) \leq \frac{1}{p_{1}} \frac{a b}{\lambda \circ \alpha(a)} x+\frac{1}{p_{2}} \frac{a b}{\lambda \circ \beta(b)} y .
$$


Since the restriction $z=T(x, \bar{a})$ of the tangent plane $z=T(x, y)$ is the tangent line to the graph $\Phi(x, \bar{a})=a \alpha^{-1}(x)$ located inside $x-z$ plane and $\alpha^{-1}$ is concave up on $\mathbb{R}_{+}$, we observe $\Phi(x, \bar{a}) \leq T(x, \bar{a})$, which holds for all $\bar{a}$. Therefore, we conclude that

$$
\Phi(x, y) \leq \theta_{1} \frac{a b}{\lambda \circ \alpha(a)} x+\theta_{2} \frac{a b}{\lambda \circ \beta(b)} y
$$

where we set $\theta_{1}:=1 / p_{1}$ and $\theta_{2}:=1 / p_{2}$.

Remark 2.4. We want to address the point that the convexity of pre-Hölder's functions is not essential in the definition of Hölder's functions, which is different from the definition of the Orlicz spaces.

The notations $\tilde{\alpha}:=\lambda \circ \alpha, \tilde{\beta}:=\lambda \circ \beta$ are used throughout the paper.

\subsection{Basic Properties of the Space $L_{\alpha}(X)$}

We now define the Lebesgue-Orlicz type function spaces $L_{\alpha}(X)$ :

$$
L_{\alpha}(X):=\left\{f: f \text { is a measurable function on } X \text { satisfying }\|f\|_{L_{\alpha}}<\infty\right\},
$$

where we set

$$
\|f\|_{L_{\alpha}}:=\tilde{\alpha}^{-1}\left(\int_{X} \tilde{\alpha}(|f(x)|) d \mu\right)
$$

Hölder-type inequality and Minkowski's inequality on the new space $L_{\alpha}(X)$ are presented as follows.

Remark 2.5. Let $\alpha$ be a Hölder's function, and let $\beta$ be the corresponding Hölder's conjugate function. Then, for any $f \in L_{\alpha}(X)$ and any $g \in L_{\beta}(X)$, we have

$$
\left|\int_{X} f(x) g(x) d \mu\right| \leq\|f\|_{L_{\alpha}}\|g\|_{L_{\beta}}
$$

and, for any $f_{1}, f_{2} \in L_{\alpha}(X)$, we have

$$
\left\|f_{1}+f_{2}\right\|_{L_{\alpha}} \leq\left\|f_{1}\right\|_{L_{\alpha}}+\left\|f_{2}\right\|_{L_{\alpha}} .
$$

The space $L_{\alpha}(X)$ is a topological vector space with inhomogeneous norm $\|\cdot\|_{L_{\alpha}}$. Furthermore, for $k \geq 0$,

$$
\left[k^{-1}\right\rceil^{-1}\|f\|_{L_{\alpha}} \leq\|k f\|_{L_{\alpha}} \leq\lceil k\rceil\|f\|_{L_{\alpha^{\prime}}}
$$


where $\lceil k\rceil$ is the ceiling of $k$, the smallest integer that is not less than $k$ (see the appendix for the proof). Also, the metric space $L_{\alpha}(X)$ is complete with respect to the metric

$$
d(f, g):=\|f-g\|_{L_{\alpha^{\prime}}} \quad \text { for } f, g \in L_{\alpha}(X)
$$

Remark 2.6. The metric space $L_{\alpha}(X)$ and the classical Orlicz space $L_{A}(X)$ differ by the convexity assumption as pointed in Remark 2.4 and by the choice of the conjugate function. In fact, for Orlicz space $L_{A}(X)$, the complementary $N$-function $\tilde{A}$ of $A$ is designed to satisfy the relation

$$
\tilde{A}^{\prime}=\left(A^{\prime}\right)^{-1}
$$

which implies, in turn,

$$
c_{1} x \leq A^{-1}(x) \tilde{A}^{-1}(x) \leq c_{2} x
$$

for some constants $c_{1}, c_{2}>0$ (see, e.g., page 265 in [1]). Hence, the conjugate relation (2.1) is devised so that the space $L_{\alpha}(X)$ contains Orlicz spaces with the $\Delta_{2}$-condition.

Whereas the Luxemburg norm for the Orlicz space $L_{A}(X)$ requires the convexity of the $N$-function $A$ for the triangle inequality of the norm, the inhomogeneous norm for the space $L_{\alpha}(X)$ does not ask the convexity of Hölder's function, and it has indeed inherited the beautiful and convenient properties from the classical Lebesgue's norm (1.4).

Here we present some remarks on the dual space of $L_{\alpha}(X)$. To each $g \in L_{\beta}(X)$ is associated a bounded linear functional $\mathcal{F}_{g}$ on $L_{\alpha}(X)$ by

$$
\mp_{g}(f):=\int_{X} f(x) g(x) d \mu
$$

and the operator (inhomogeneous) norm of $\mathcal{F}_{g}$ is at most $\|g\|_{L_{\beta}}$ :

$$
\left\|\mathcal{F}_{g}\right\|_{L_{\alpha}^{\prime}}:=\sup \left\{\frac{\left|\int_{X} f g d \mu\right|}{\|f\|_{L_{\alpha}}}: f \in L_{\alpha}(X), f \neq 0\right\} \leq\|g\|_{L_{\beta}} .
$$

For $0 \neq g \in L_{\beta}(X)$, if we put $f(x):=\tilde{\beta}(|g(x)|) \operatorname{sgn}(g(x)) /|g(x)|$, then we have that $f \in L_{\alpha}(X)$ and

$$
\left\|\mathscr{F}_{g}\right\|_{L_{\alpha}^{\prime}}=\sup \left\{\frac{\left|\int_{X} f g d \mu\right|}{\|f\|_{L_{\alpha}}}: f \in L_{\alpha}(X), f \neq 0\right\} \geq \frac{\left|\int_{X} f g d \mu\right|}{\|f\|_{L_{\alpha}}}=\|g\|_{L_{\beta}} .
$$

This implies that the mapping $g \mapsto \mathcal{F}_{g}$ is isometric from $L_{\beta}(X)$ into the space of continuous linear functionals $L_{\alpha}(X)^{\prime}$. Furthermore, it can be shown that the linear transformation $\mathcal{F}$ : $L_{\beta}(X) \rightarrow L_{\alpha}(X)^{\prime}$ is onto the following. 
Remark 2.7 (dual space of $L_{\alpha}(X)$ ). Let $\beta$ be the conjugate Hölder's function of a Hölder's function $\alpha$. Then the dual space $L_{\alpha}(X)^{\prime}$ is isometrically isomorphic to $L_{\beta}(X)$.

\subsection{Sobolev-Type Space $W_{\alpha}^{1}$}

Let $\Omega$ be an open subset of $\mathbb{R}^{n}$. The Sobolev-type space $W_{\alpha}^{1}(\Omega)$ is employed by

$$
W_{\alpha}^{1}(\Omega):=\left\{u \in L_{\alpha}(\Omega) \mid \partial_{x_{j}} u \in L_{\alpha}(\Omega), j=1,2, \ldots, n\right\}
$$

together with the norm

$$
\|u\|_{W_{\alpha}^{1}}:=\|u\|_{L_{\alpha}}+\tilde{\alpha}^{-1}\left(\sum_{j=1}^{n} \tilde{\alpha}\left(\left\|\partial_{x_{j}} u\right\|_{L_{\alpha}}\right)\right)
$$

where $\partial_{x_{j}}:=\partial / \partial x_{j}$. Then it can be shown that the function space $W_{\alpha}^{1}(\Omega)$ is a separable complete metric space and $C^{\infty}(\Omega) \cap W_{\alpha}^{1}(\Omega)$ is dense in $W_{\alpha}^{1}(\Omega)$. The proofs are very similar to the case of Orlicz spaces (see page 274 in [1]).

The completion of the space $C_{c}^{\infty}(\Omega)$ with respect to the norm $\|\cdot\|_{W_{\alpha}^{1}}$ is denoted by $W_{\alpha, 0}^{1}(\Omega)$, where $C_{c}^{\infty}(\Omega)$ is the space of smooth functions with compact support.

We are in the position of introducing the trace operator and Poincarés inequality on $W_{\alpha}^{1}(\Omega)$, which are important by themselves and also useful for the proof of the existence theorem. We say that a pre-Hölder function $\beta$ is to satisfy a slope condition if there exists some positive constant $c>1$ for which

$$
\tilde{\beta}^{\prime}(x) \geq c \frac{\tilde{\beta}(x)}{x}
$$

holds for almost every $x>0$. The slope condition (2.32), in fact, corresponds to the $\Delta_{2}$ condition for Orlicz spaces (page 266 in [1]).

The boundary trace on $C_{c}^{\infty}(\bar{\Omega})$ can be extended to the space $W_{\alpha}^{1}(\Omega)$ as follows. For the case $\Omega=\mathbb{R}_{+}^{n}:=\left\{\left(x^{\prime}, x_{n}\right): x^{\prime} \in \mathbb{R}^{n-1}, x_{n}>0\right\}$ and for a smooth function $\phi \in C_{c}^{\infty}\left(\overline{\mathbb{R}_{+}^{n}}\right)$, we observe

$$
\begin{aligned}
\tilde{\alpha}\left(\left|\phi\left(x^{\prime}, 0\right)\right|\right) & =-\int_{0}^{\infty} \partial_{x_{n}} \tilde{\alpha}\left(\left|\phi\left(x^{\prime}, x_{n}\right)\right|\right) d x_{n} \\
& \leq \int_{0}^{\infty} \tilde{\alpha}^{\prime}\left(\left|\phi\left(x^{\prime}, x_{n}\right)\right|\right)\left|\partial_{x_{n}} \phi\left(x^{\prime}, x_{n}\right)\right| d x_{n} \\
& \leq\left\|\partial_{x_{n}} \phi\left(x^{\prime}, \cdot\right)\right\|_{L_{\alpha}(0, \infty)}\left\|\tilde{\alpha}^{\prime}\left(\left|\phi\left(x^{\prime}, \cdot\right)\right|\right)\right\|_{L_{\beta}(0, \infty)} .
\end{aligned}
$$

Owing to the identity (2.6), we have

$$
\tilde{\alpha}^{\prime}(t)=s+t \frac{\alpha^{\prime}(t)}{\beta^{\prime}(s)}, \quad s=\frac{\tilde{\alpha}(t)}{t} .
$$


On the other hand, we can notice that the slope condition (2.32) is equivalent to saying

$$
\beta^{\prime}\left(\frac{\tilde{\alpha}(t)}{t}\right) \geq \frac{c}{\lambda^{\prime}(\alpha(t))} t
$$

Reflecting this to the identity (2.34), we have

$$
\tilde{\alpha}^{\prime}(|\phi(x)|) \leq \frac{c}{c-1} \frac{\tilde{\alpha}(|\phi(x)|)}{|\phi(x)|}, \quad x=\left(x^{\prime}, x_{n}\right) .
$$

Therefore, we have

$$
\begin{aligned}
\tilde{\beta}^{-1}\left(\int_{0}^{\infty} \tilde{\beta}\left(\tilde{\alpha}^{\prime}\left(\left|\phi\left(x^{\prime}, x_{n}\right)\right|\right)\right) d x_{n}\right) & \leq \tilde{\beta}^{-1}\left(\int_{0}^{\infty} \tilde{\beta}\left(\frac{c}{c-1} \frac{\tilde{\alpha}\left(\left|\phi\left(x^{\prime}, x_{n}\right)\right|\right)}{\left|\phi\left(x^{\prime}, x_{n}\right)\right|}\right) d x_{n}\right) \\
& \leq\left[\frac{c}{c-1}\right] \tilde{\beta}^{-1}\left(\int_{0}^{\infty} \tilde{\beta}\left(\frac{\tilde{\alpha}\left(\left|\phi\left(x^{\prime}, x_{n}\right)\right|\right)}{\left|\phi\left(x^{\prime}, x_{n}\right)\right|}\right) d x_{n}\right) \\
& =\left[\frac{c}{c-1}\right] \tilde{\beta}^{-1}\left(\int_{0}^{\infty} \tilde{\alpha}\left(\left|\phi\left(x^{\prime}, x_{n}\right)\right|\right) d x_{n}\right) .
\end{aligned}
$$

Inserting this into the right side of (2.33), we obtain

$$
\begin{aligned}
\tilde{\alpha}\left(\left|\phi\left(x^{\prime}, 0\right)\right|\right) & \leq C\left\|\partial_{x_{n}} \phi\left(x^{\prime}, \cdot\right)\right\|_{L_{\alpha}(0, \infty)} \tilde{\beta}^{-1} \circ \tilde{\alpha}\left(\left\|\phi\left(x^{\prime}, \cdot\right)\right\|_{L_{\alpha}(0, \infty)}\right) \\
& \leq C\left[\widetilde{\alpha}\left(\left\|\partial_{x_{n}} \phi\left(x^{\prime}, \cdot\right)\right\|_{L_{\alpha}(0, \infty)}\right)+\tilde{\alpha}\left(\left\|\phi\left(x^{\prime}, \cdot\right)\right\|_{L_{\alpha}(0, \infty)}\right)\right]
\end{aligned}
$$

for some positive constant $C$. The comparable condition (2.12) has been used in the second inequality. Taking integration on both sides over $\mathbb{R}^{n-1}$, we obtain

$$
\tilde{\alpha}\left(\|\phi\|_{L_{\alpha}\left(\mathbb{R}^{n-1}\right)}\right) \leq C\left[\tilde{\alpha}\left(\left\|\partial_{x_{n}} \phi\right\|_{L_{\alpha}\left(\mathbb{R}_{+}^{n}\right)}\right)+\tilde{\alpha}\left(\|\phi\|_{L_{\alpha}\left(\mathbb{R}_{+}^{n}\right)}\right)\right]
$$

This inequality says that the trace on $C_{c}^{\infty}\left(\overline{\mathbb{R}_{+}^{n}}\right)$ can be uniquely extended to the space $W_{\alpha}^{1}\left(\mathbb{R}_{+}^{n}\right)$ as a metric space. For the case $\Omega$ being a bounded open subset ( $\Omega$ can be more general, e.g., it permits unbounded domains satisfying the uniform $C^{m}$-regularity condition (page 84 in [1])), the partitions of unity can be employed to turn the case locally into that of $\mathbb{R}_{+}^{n}$ with appropriate Jacobians. Gluing a finite number of estimates (2.39), we get the following proposition (for details, see page 164 in [1] or page 56 in [2]).

Proposition 2.8 (Trace map on $\left.W_{\alpha}^{1}\right)$. Let $(\alpha, \beta)$ be a Hölder pair obeying the slope condition (2.32), and let $\Omega$ be a bounded open set with smooth boundary in $\mathbb{R}^{n}$. Then the trace operator $\gamma: W_{\alpha}^{1}(\Omega) \rightarrow$ $L_{\alpha}(\partial \Omega)$ is continuous and uniquely determined by $\gamma(u)=\left.u\right|_{\partial \Omega}$ on those $u \in C_{c}^{\infty}(\bar{\Omega})$.

We present Poincarés inequality whose proof can be found in the appendix. 
Proposition 2.9 (Poincare's inequality). Let $(\alpha, \beta)$ be a Hölder pair with the slope condition (2.32), and let $\Omega$ be an open set in $\mathbb{R}^{n}$ which is bounded in some direction; that is, there is a vector $v \in \mathbb{R}^{n}$ such that

$$
\sup \{|x \cdot v|: x \in \Omega\}<\infty
$$

Then there is a constant $C>0$ such that, for any $f \in W_{\alpha}^{1}(\Omega)$ with $f(x)=0$ (in the sense of the trace map) for $x \in \partial \Omega$ and $x \cdot v \neq 0$,

$$
\|f\|_{L_{\alpha}} \leq C\|v \cdot \nabla f\|_{L_{\alpha}}
$$

\section{Nonlinear Elliptic Equations of General Flux Terms}

In this section $\Omega$ is a fixed bounded open set in $\mathbb{R}^{n}$ with smooth boundary. We are concerned with an elliptic partial differential equation:

$$
-\nabla \cdot \mathbf{J}(u)=f
$$

where the flux vector field is given by

$$
\mathrm{J}(u):=\left(\frac{\tilde{\alpha}\left(\left|\partial_{x_{1}} u\right|\right)}{\partial_{x_{1}} u}, \frac{\tilde{\alpha}\left(\left|\partial_{x_{2}} u\right|\right)}{\partial_{x_{2}} u}, \ldots, \frac{\tilde{\alpha}\left(\left|\partial_{x_{n}} u\right|\right)}{\partial_{x_{n}} u}\right) .
$$

We look for solutions of the elliptic equation (3.1) on an appropriate space. In fact, the function space that can permit solutions of (3.1) turns out to be the space $W_{\alpha, 0}^{1}(\Omega):=V$.

Now, we state the existence theorem of the nonlinear elliptic equation with general flux term (3.1).

Theorem 3.1. Let $(\alpha, \beta)$ be a Hölder pair satisfying the slope condition (2.32). Then, for any functional $f \in V^{\prime}$, there exists a solution $u \in V$ satisfying the elliptic partial differential equation

$$
-\sum_{j=1}^{n} \frac{\partial}{\partial x_{j}}\left(\frac{\tilde{\alpha}\left(\left|u_{x_{j}}\right|\right)}{u_{x_{j}}}\right)=f
$$

We start to set up the functional equation associated with (3.1). Let $\phi \in C_{c}^{\infty}(\Omega)$. Then we have

$$
-\int_{\Omega} \nabla \cdot \mathbf{J}(\nabla u) \phi d \mu=\int_{\Omega} f \phi d \mu
$$

Then, by Gauss-Green theorem, the left-hand side becomes

$$
-\int_{\Omega} \nabla \cdot(\mathbf{J}(\nabla u)) \phi d \mu=\int_{\Omega} \mathbf{J}(\nabla u) \cdot \nabla \phi d \mu=\int_{\Omega} \sum_{j=1}^{n} \frac{\tilde{\alpha}\left(\left|\partial_{x_{j}} u\right|\right)}{\partial_{x_{j}} u} \partial_{x_{j}} \phi d \mu .
$$


We will consider the operator $\mathbb{A}$ defined by

$$
\mathcal{A} u(\phi):=\sum_{j=1}^{n} \int_{\Omega} \frac{\tilde{\alpha}\left(\left|u_{x_{j}}\right|\right)}{u_{x_{j}}} \phi_{x_{j}} d \mu=\sum_{j=1}^{n} \int_{\Omega} \beta^{-1} \circ \alpha\left(\left|u_{x_{j}}\right|\right) \phi_{x_{j}} d \mu,
$$

for $u, v \in V:=W_{\alpha, 0}^{1}(\Omega)$. We investigate some properties of the operator $\mathcal{A}: V \rightarrow V^{\prime}$ which will be used for the proof of the existence theorem.

Lemma 3.2. One has an estimate: for $u \in V$,

$$
|\mathcal{A} u(\phi)| \leq \beta^{-1} \circ \alpha\left(\|u\|_{W_{\alpha}^{1}}\right) \cdot\|\phi\|_{W_{\alpha}^{1}} \text {. }
$$

In particular, the operator $A: V \rightarrow V^{\prime}$ is bounded; that is, for any bounded set $S$ in $V$, the image $\mathcal{A}(S)$ of $S$ is bounded in $V^{\prime}$.

Proof. By Hölder's inequality and identity (2.2), we have

$$
\begin{aligned}
|\mathcal{A} u(\phi)| & \leq \sum_{j=1}^{n}\left|\int_{\Omega} \frac{\tilde{\alpha}\left(\left|\partial_{x_{j}} u\right|\right)}{\partial_{x_{j}} u} \partial_{x_{j}} \phi d \mu\right| \\
& \leq \sum_{j=1}^{n} \tilde{\beta}^{-1}\left(\int_{\Omega} \tilde{\beta}\left(\frac{\tilde{\alpha}\left(\left|\partial_{x_{j}} u\right|\right)}{\left|\partial_{x_{j}} u\right|}\right) d \mu\right) \tilde{\alpha}^{-1}\left(\int_{\Omega} \tilde{\alpha}\left(\left|\partial_{x_{j}} \phi\right|\right) d \mu\right) \\
& =\sum_{j=1}^{n} \tilde{\beta}^{-1}\left(\int_{\Omega} \tilde{\alpha}\left(\left|\partial_{x_{j}} u\right|\right) d \mu\right)\left\|\partial_{x_{j}} \phi\right\|_{L_{\alpha}} \\
& =\sum_{j=1}^{n} \tilde{\beta}^{-1} \circ \tilde{\alpha}\left(\left\|\partial_{x_{j}} u\right\|_{L_{\alpha}}\right)\left\|\partial_{x_{j}} \phi\right\|_{L_{\alpha}} .
\end{aligned}
$$

Hölder's inequality with respect to the counting measure reads as for $a_{j}, b_{j}>0$

$$
\sum_{j=1}^{n} a_{j} b_{j} \leq \tilde{\alpha}^{-1}\left(\sum_{j=1}^{n} \tilde{\alpha}\left(a_{j}\right)\right) \tilde{\beta}^{-1}\left(\sum_{j=1}^{n} \tilde{\beta}\left(b_{j}\right)\right) .
$$

Apply this inequality to (3.8), and we get

$$
\begin{aligned}
|\mathcal{A} u(\phi)| & \leq \tilde{\beta}^{-1}\left(\sum_{j=1}^{n} \tilde{\alpha}\left(\left\|\partial_{x_{j}} u\right\|_{L_{\alpha}}\right)\right) \tilde{\alpha}^{-1}\left(\sum_{j=1}^{n} \tilde{\alpha}\left(\left\|\partial_{x_{j}} \phi\right\|_{L_{\alpha}}\right)\right) \\
& \leq \beta^{-1} \circ \alpha\left(\|u\|_{W_{\alpha}^{1}}\right) \cdot\|\phi\|_{W_{\alpha}^{1}} .
\end{aligned}
$$

Since $\alpha$ and $\beta^{-1}$ are continuous on $\mathbb{R}_{+}$, the operator $\mathcal{A}$ is bounded. 
Lemma 3.3. The operator $A: V \rightarrow V^{\prime}$ is continuous.

Proof. For each $1 \leq i \leq n$, we have $\left\|u_{x_{i}}\right\|_{L_{\alpha}} \leq\|u\|_{W_{\alpha}^{1}}$. Hence, the operator $\partial / \partial x_{i}: W_{\alpha}^{1}(\Omega) \rightarrow$ $L_{\alpha}(\Omega)$ is continuous. Hence, we define an operator $T: L_{\alpha}(\Omega) \rightarrow L_{\beta}(\Omega)$ by $T(u)=\widetilde{\alpha}(|u|) / u$ for $u \neq 0$ and $T(0)=0$. Then the operator $T$ is well defined. Indeed, for $u \in L_{\alpha}$, we have

$$
\int_{\Omega} \tilde{\beta}(|T(u)|) d \mu=\int_{\Omega} \tilde{\beta}\left(\frac{\tilde{\alpha}(|u|)}{|u|}\right) d \mu=\int_{\Omega} \tilde{\alpha}(|u|) d \mu<\infty
$$

This says that $\|T(u)\|_{L_{\beta}}=\beta^{-1} \circ \alpha\left(\|u\|_{L_{\alpha}}\right)$, which implies the continuity of $T$.

Define $\left(\partial / \partial x_{i}\right)^{*}: L_{\beta} \rightarrow V^{\prime}$ by

$$
\left(\frac{\partial}{\partial x_{i}}\right)^{*} v(\phi):=\int_{\Omega} v(x) \phi_{x_{i}}(x) d x
$$

for $v \in L_{\beta}$ and $\phi \in V$. Then the operator $\left(\partial / \partial x_{i}\right)^{*}$ is continuous, since

$$
\left\|\left(\frac{\partial}{\partial x_{i}}\right)^{*} v\right\|_{V^{\prime}}:=\sup _{\|\phi\|_{V} \neq 0} \frac{\left|\int_{\Omega} v(x) \phi_{x_{i}}(x) d x\right|}{\|\phi\|_{V}} \leq \sup _{\|\phi\|_{V} \neq 0} \frac{\|v\|_{L_{\beta}}\left\|\phi_{x_{i}}\right\|_{L_{\alpha}}}{\|\phi\|_{V}} \leq\|v\|_{L_{\beta}} .
$$

Therefore, the composition maps $S_{j}:=\left(\partial / \partial x_{j}\right)^{*} \circ T \circ\left(\partial / \partial x_{j}\right): V \rightarrow V^{\prime}, j=1,2, \ldots, n$, are continuous. Since the operator $A$ is just a linear combination of the operators $S_{j}$, we have the continuity of $\mathcal{A}$.

For $a, b>0$, we have

$$
\left(\frac{\tilde{\alpha}(a)}{a}-\frac{\tilde{\alpha}(b)}{b}\right)(a-b)=\left(\beta^{-1} \circ \alpha(a)-\beta^{-1} \circ \alpha(b)\right)(a-b)>0,
$$

from the fact that $\beta^{-1} \circ \alpha$ is monotone increasing. This implies the following.

Lemma 3.4. The operator $A$ is monotone, that is,

$$
(\mathcal{A} u-\mathcal{A} v)(u-v)>0 \quad \text { for } u, v \in V \text {. }
$$

Proof. By the above computation, we get

$$
(\mathcal{A} u-\mathscr{A} v)(u-v)=\sum_{j=1}^{n} \int_{\Omega}\left(\frac{\tilde{\alpha}\left(\left|u_{x_{j}}\right|\right)}{u_{x_{j}}}-\frac{\tilde{\alpha}\left(\left|v_{x_{j}}\right|\right)}{v_{x_{j}}}\right)\left(u_{x_{j}}-v_{x_{j}}\right) d x>0
$$

for $u, v \in V$. 
We say that an operator $\mathcal{A}: V \rightarrow V^{\prime}$ is coercive if

$$
\lim _{\|u\|_{V} \rightarrow \infty} \frac{\mathcal{A} u(u)}{\|u\|_{V}}=\infty
$$

Remark 3.5. Coercivity of $\mathcal{A}$ implies that for any $f \in V^{\prime}$, there is $N>0$ such that

$$
f(u)<A u(u),
$$

provided that $\|u\|_{V} \geq N$. In fact, the limit (3.17) says that, for any $M>0$, there exists a positive integer $N$ such that $\mathcal{A} u(u)>M\|u\|_{V}$ for $\|u\|_{V} \geq N$. Hence, in particular, by taking $M:=\|f\|_{V^{\prime}}$, we have

$$
f(u) \leq|f(u)| \leq\|f\|_{V^{\prime}}\|u\|_{V}<\mathcal{A} u(u),
$$

if $\|u\|_{V} \geq N$.

In the following lemma, we assume that the Hölder pair $(\alpha, \beta)$ permits the slope condition (2.32).

Lemma 3.6. The operator $A: V \rightarrow V^{\prime}$ is coercive.

Proof. By virtue of Poincaré's inequality, we see that

$$
\|u\|_{L_{\alpha}} \leq C \tilde{\alpha}^{-1}\left(\int_{\Omega} \tilde{\alpha}\left(\left|u_{x_{1}}\right|\right) d x\right) \leq C \tilde{\alpha}^{-1}\left(\sum_{j=1}^{n} \int_{\Omega} \tilde{\alpha}\left(\left|u_{x_{j}}\right|\right) d x\right)
$$

for some positive constant $C$. Then we have

$$
\|u\|_{V}=\|u\|_{L_{\alpha}}+\tilde{\alpha}^{-1}\left(\sum_{j=1}^{n} \int_{\Omega} \tilde{\alpha}\left(\left|u_{x_{j}}\right|\right) d x\right) \leq(C+1) \tilde{\alpha}^{-1}\left(\sum_{j=1}^{n} \int_{\Omega} \tilde{\alpha}\left(\left|u_{x_{j}}\right|\right) d x\right) .
$$

Hence, as $\|u\|_{V} \rightarrow \infty$, we observe $\sum_{j=1}^{n} \int_{\Omega} \tilde{\alpha}\left(\left|u_{x_{j}}\right|\right) d x \rightarrow \infty$. Therefore, we get

$$
\begin{aligned}
\frac{\mathscr{A} u(u)}{\|u\|_{V}} & \geq \frac{\sum_{j=1}^{n} \int_{\Omega} \tilde{\alpha}\left(\left|u_{x_{j}}\right|\right) d x}{(C+1) \tilde{\alpha}^{-1}\left(\sum_{j=1}^{n} \int_{\Omega} \tilde{\alpha}\left(\left|u_{x_{j}}\right|\right) d x\right)} \\
& =\frac{1}{C+1} \tilde{\beta}^{-1}\left(\sum_{j=1}^{n} \int_{\Omega} \tilde{\alpha}\left(\left|u_{x_{j}}\right|\right) d x\right) \rightarrow \infty,
\end{aligned}
$$

as $\|u\|_{V} \rightarrow \infty$.

We now present the proof of the main theorem. 
Proof of Theorem 3.1. We note that $V=\left\{u \in W_{\alpha}^{1}(\Omega) \mid u=0\right.$ on $\left.\partial \Omega\right\}$ is a separable reflexive complete metric space. Hence, we can choose an independent set of vectors $\left\{w_{1}, w_{2}, \ldots\right\}$ whose linear spans are dense in $V$. For each $m \geq 1$, we denote by $V_{m}$ the subspace of $V$ spanned by the set of vectors $\left\{w_{1}, w_{2}, \ldots, w_{m}\right\}$, that is, $V_{m}:=\operatorname{span}\left\{w_{1}, w_{2}, \ldots, w_{m}\right\}$, and we define the natural (vector space) isomorphism $j_{m}: V_{m} \rightarrow \mathbb{R}^{m}$ by

$$
\sum_{i=1}^{m} a_{i} w_{i} \longmapsto\left(a_{1}, a_{2}, \ldots, a_{m}\right)
$$

Note that $j_{m}^{-1}: \mathbb{R}^{m} \rightarrow V_{m}$ is continuous, because it is a combination of finitely many scalar multiplications and additions each of which is continuous. Hence, by denoting the inclusion map from $V_{m}$ to $V$ by $i_{m}: V_{m} \rightarrow V$, we have $\pi_{m}=i_{m} \circ j_{m}^{-1}: \mathbb{R}^{m} \rightarrow V$ is continuous.

We define

$$
\mathcal{A} u(\phi)=\sum_{j=1}^{n} \int_{\Omega} \frac{\tilde{\alpha}\left(\left|u_{x_{j}}\right|\right)}{u_{x_{j}}} \phi_{x_{j}} d x=\sum_{j=1}^{n} \int_{\Omega} \beta^{-1} \circ \alpha\left(\left|u_{x_{j}}\right|\right) \phi_{x_{j}} d x,
$$

for $u, \phi \in V$. For fixed $m \in \mathbb{N}$, we will find a solution $u_{m} \in V_{m}$ for a system

$$
\mathcal{A}\left(u_{m}\right)\left(w_{j}\right)=f\left(w_{j}\right), \quad 1 \leq j \leq m
$$

To accomplish it, we first show that $\pi_{m}^{*} \circ \mathcal{A} \circ \pi_{m}: \mathbb{R}^{m} \rightarrow\left(\mathbb{R}^{m}\right)^{\prime}$ is continuous, where we set $\pi_{m}^{*}(\phi)(x):=\phi\left(\pi_{m}(x)\right)$, for $\phi \in V^{\prime}, x \in \mathbb{R}^{m}$. Indeed, the nonlinear operator $\mathscr{A}: V \rightarrow V^{\prime}$ is continuous by Lemma 3.3, and the dual linear operator $\pi_{m}^{*}: V^{\prime} \rightarrow\left(\mathbb{R}^{m}\right)^{\prime}$ of $\pi_{m}$ is continuous.

Now we define a map $F_{m}: \mathbb{R}^{m} \rightarrow\left(\mathbb{R}^{m}\right)^{\prime}$ by

$$
F_{m}(v):=\pi_{m}^{*} \circ \mathcal{A} \circ \pi_{m}(v)-\pi_{m}^{*}(f)
$$

for all $v \in \mathbb{R}^{m}$. We will show that $F_{m}$ has a root. By Lemma 3.6 and Remark 3.5, there is a positive number $N>0$ such that for any $u$ with $\|u\|_{V} \geq N$, we have

$$
A u(u)>f(u)
$$

Hence, for any $\left\|\pi_{m}(v)\right\|_{V} \geq N$, we obtain

$$
\begin{aligned}
F_{m}(v)(v) & =\pi_{m}^{*} \circ \mathcal{A} \circ \pi_{m}(v)(v)-\pi_{m}^{*} f(v) \\
& =\mathcal{A}\left(\pi_{m}(v)\right)\left(\pi_{m}(v)\right)-f\left(\pi_{m}(v)\right)>0 .
\end{aligned}
$$

From the fact that $\lim _{|v| \rightarrow \infty} \pi_{m}(v)=\infty$, there is $r>0$ such that $|v| \geq r$ implies $\left\|\pi_{m}(v)\right\|_{V} \geq N$. Therefore, by letting $\mathcal{R}: \mathbb{R}^{m} \rightarrow\left(\mathbb{R}^{m}\right)^{\prime}$ the Riesz map and putting $\widetilde{F}_{m}:=\mathcal{R}^{-1} \circ F_{m}$, we obtain

$$
\tilde{F}_{m}(v) \cdot v=F_{m}(v)(v)>0, \quad \text { if }|v| \geq r
$$


Together with the continuity of $\widetilde{F}_{m}$, we know that $\widetilde{F}_{m}$ has a root inside the ball $\left\{x \in \mathbb{R}^{m}\right.$ : $|x| \leq r\}$ by virtue of Brouwer's fixed point theorem. Hence, $F_{m}$ has a root $F_{m}\left(\bar{u}_{m}\right)=0$ for some $\left|\bar{u}_{m}\right| \leq r$. Therefore, for each $m \geq 1$, we have

$$
\left(\mathcal{A} \circ \pi_{m}\left(\bar{u}_{m}\right)\right)\left(\pi_{m}(v)\right)-f\left(\pi_{m}(v)\right)=0 \quad \forall v \in \mathbb{R}^{m},
$$

or equivalently

$$
\left(\mathcal{A} \circ \pi_{m}\left(\bar{u}_{m}\right)\right)(w)-f(w)=0 \quad \forall w \in V_{m} .
$$

Denote $\pi_{m}\left(\bar{u}_{m}\right):=u_{m}$, and we get

$$
\mathcal{A} u_{m}=f \text { in } V_{m}^{\prime} \text {. }
$$

It follows from (3.28) that $\left\|u_{m}\right\|_{V} \leq N$. We also have that the sequence $\left\{\left\|\mathcal{A} u_{m}\right\|_{V^{\prime}}\right\}$ is bounded since the operator $A$ is bounded (Lemma 3.2). Thus, the sequence $\left\{\left|f\left(u_{m}\right)\right|\right\}$ is bounded. Then there exists a subsequence $\left\{u_{m_{k}}\right\}$ of $\left\{u_{m}\right\}$ and an element $u$ in $V$ such that

(i) $u_{m_{k}} \rightarrow u$ in $V$,

(ii) $\mathcal{A} u_{m_{\mathrm{k}}} \rightarrow f$ in $V^{\prime}$ (by (3.32)),

(iii) $\left(\mathcal{A} u_{m_{k}}\right)\left(u_{m_{k}}\right)=f\left(u_{m_{k}}\right) \rightarrow f(u)$ (by the weak continuity of $f$ ).

Since $\mathcal{A}$ is monotone (Lemma 3.4), we have that, for all $v \in V$,

$$
\left(\mathcal{A} u_{m_{k}}\right)\left(u_{m_{k}}\right)-\left(\mathcal{A} u_{m_{k}}\right)(v)-(\mathcal{A} v)\left(u_{m_{k}}\right)+(\mathcal{A} v)(v) \geq 0
$$

Then take the limit as $k \rightarrow \infty$, and we get $f(u)-f(v)-(\mathcal{A v})(u)+(\mathcal{A v})(v) \geq 0$. This is equivalent to

$$
(f-\mathcal{A} v)(u-v) \geq 0
$$

and this holds for all $v \in V$. Now take $v:=u-t w$ for any $w \in V$ and any $t>0$, then plug $v$ into (3.34) to have $t(f-\mathcal{A}(u-t w)) w \geq 0$, or $f(w)-\mathcal{A}(u-t w) w \geq 0$. By the continuity of $\mathcal{A}$ (Lemma 3.3), we let $t \rightarrow 0$ to obtain

$$
f(w)-\mathcal{A}(u) w \geq 0,
$$

for all $w \in V$. We replace $w$ in (3.35) with $-w$ to find

$$
(f-\mathcal{A}(u))(w) \leq 0 .
$$

Combine (3.35) with (3.36), and we finally obtain

$$
f=\mathcal{A}(u) .
$$

The proof is now completed. 


\section{Appendix}

We present the proof of Proposition 2.9. We first set up the following lemma.

Lemma A.1. The scalar multiplication on $L_{\alpha}(X)$ over $\mathbb{R}$ is continuous. Furthermore, for $k>0$,

$$
\left\lceil k^{-1}\right\rceil^{-1}\|f\|_{L_{\alpha}} \leq\|k f\|_{L_{\alpha}} \leq\lceil k\rceil\|f\|_{L_{\alpha^{\prime}}}
$$

where $\lceil k\rceil$ is the ceiling of $k$, the smallest integer that is not less than $k$.

Proof. The monotonicity of $\alpha, \alpha^{-1}$ and Minkowski's inequality deliver

$$
\|k f\|_{L_{\alpha}} \leq\|\lceil k\rceil f\|_{L_{\alpha}} \leq\lceil k\rceil\|f\|_{L_{\alpha}}
$$

Hence, $f_{n} \rightarrow f$ in $L_{\alpha}(X)$ implies $k f_{n} \rightarrow k f$ in $L_{\alpha}(X)$. In turn, it leads to $\|f\|_{L_{\alpha}}=$ $\|(1 / k) k f\|_{L_{\alpha}} \leq[1 / k\rceil\|k f\|_{L_{\alpha^{\prime}}}$ and so $\lceil 1 / k\rceil^{-1}\|f\|_{L_{\alpha}} \leq\|k f\|_{L_{\alpha}}$. Now, it remains to check that the conditions $k_{n} \rightarrow k$ and $f \in L_{\alpha}(X)$ imply $k_{n} f \stackrel{\rightarrow}{\rightarrow} k f$ in $L_{\alpha}(X)$. Indeed, the inequality (A.2) shows implicitly that the sequence $\left\{\alpha\left(\left|\left(k_{n}-k\right) f\right|\right)\right\}$ is dominated by an $L_{\alpha}$-function $\alpha(2 M|f|)$ with $M:=\left[\max _{n \in \mathbb{N}}\left|k_{n}\right| \mid\right.$; hence, by Lebesgue-dominated convergence theorem, we have

$$
\lim _{n \rightarrow \infty} \int_{X} \tilde{\alpha}\left(\left|\left(k_{n}-k\right) f\right|\right) d \mu=\int_{X} \lim _{n \rightarrow \infty} \tilde{\alpha}\left(\left|\left(k_{n}-k\right) f(x)\right|\right) d \mu=0 .
$$

This gives the desired convergence.

We now return to prove Proposition 2.9.

Proof of Proposition 2.9. Without loss of generality, we may assume that $v=(1,0, \ldots, 0)$. For $\phi \in C_{c}^{\infty}(\Omega)$,

$$
\partial_{x_{1}}\left(x_{1} \tilde{\alpha}(|\phi(x)|)\right)=\tilde{\alpha}(|\phi(x)|)+x_{1} \tilde{\alpha}^{\prime}(|\phi(x)|)(\operatorname{sgn} \phi(x))\left(\partial_{x_{1}} \phi(x)\right) .
$$

Integration of both sides yields

$$
\int_{\Omega} \partial_{x_{1}}\left(x_{1} \tilde{\alpha}(|\phi(x)|)\right) d x=\int_{\Omega} \tilde{\alpha}(|\phi(x)|) d x+\int_{\Omega} x_{1} \tilde{\alpha}^{\prime}(|\phi(x)|)(\operatorname{sgn} \phi(x))\left(\partial_{x_{1}} \phi(x)\right) d x .
$$

The boundary condition makes the left-hand side zero: $\int_{\Omega} \partial_{x_{1}}\left(x_{1} \tilde{\alpha}(|\phi(x)|)\right) d x=0$. Then, by virtue of Hölder's inequality, identity (A.5) becomes

$$
\int_{\Omega} \tilde{\alpha}(|\phi(x)|) d x \leq k \tilde{\beta}^{-1}\left(\int_{\Omega} \tilde{\beta}\left(\left|\tilde{\alpha}^{\prime}(|\phi(x)|)\right|\right) d x\right) \tilde{\alpha}^{-1}\left(\int_{\Omega} \tilde{\alpha}\left(\left|\partial_{x_{1}} \phi(x)\right|\right) d x\right),
$$


where $k=\sup \{|x \cdot v|: x \in \Omega\}$. The slope condition (2.35) together with the identity (2.34) and the argument presented at Section 2.2 yield

$$
\begin{aligned}
\tilde{\beta}^{-1}\left(\int_{\Omega} \tilde{\beta}\left(\tilde{\alpha}^{\prime}(|\phi(x)|)\right) d x\right) & \leq \tilde{\beta}^{-1}\left(\int_{\Omega} \tilde{\beta}\left(\frac{c}{c-1} \frac{\tilde{\alpha}(|\phi(x)|)}{|\phi(x)|}\right) d x\right) \\
& =\left[\frac{c}{c-1}\right] \tilde{\beta}^{-1}\left(\int_{\Omega} \tilde{\alpha}(|\phi(x)|) d x\right) .
\end{aligned}
$$

Therefore, from the estimate (A.6), we conclude that

$$
\tilde{\alpha}\left(\|\phi\|_{L_{\alpha}}\right) \leq C \beta^{-1} \circ \alpha\left(\|\phi\|_{L_{\alpha}}\right)\left\|\partial_{x_{1}} \phi\right\|_{L_{\alpha^{\prime}}}
$$

or equivalently

$$
\frac{\tilde{\alpha}\left(\|\phi\|_{L_{\alpha}}\right)}{\beta^{-1} \circ \alpha\left(\|\phi\|_{L_{\alpha}}\right)} \leq C\left\|\partial_{x_{1}} \phi\right\|_{L_{\alpha^{\prime}}}
$$

for some constant $C$. Identity (2.3) leads to $\|\phi\|_{L_{\alpha}} \leq C\left\|\partial_{x_{1}} \phi\right\|_{L_{\alpha}}$. The density argument gives the desired inequality.

\section{Acknowledgment}

The author was supported by the research fund of Dankook University in 2009.

\section{References}

[1] R. A. Adams and J. J. F. Fournier, Sobolev Spaces, vol. 140 of Pure and Applied Mathematics, Academic Press, Amsterdam, The Netherlands, 2nd edition, 2003.

[2] R. E. Showalter, Monotone Operators in Banach Space and Nonlinear Partial Differential Equations, vol. 49 of Mathematical Surveys and Monographs, AMS, 1997. 